\title{
On Multi-Laplace Transform for Solving Nonlinear Partial Differential Equations with Mixed Derivatives
}

\author{
Abdon Atangana ${ }^{1}$ and Suares Clovis Oukouomi Noutchie ${ }^{2}$ \\ ${ }^{1}$ Institute for Groundwater Studies, Faculty of Natural and Agricultural Sciences, University of the Free State, \\ Bloemfontein 9300, South Africa \\ ${ }^{2}$ Department of Mathematical Sciences, North-West University, Mafikeng Campus, Mmabatho 2735, South Africa
}

Correspondence should be addressed to Abdon Atangana; abdonatangana@yahoo.fr

Received 28 April 2014; Accepted 21 May 2014; Published 11 June 2014

Academic Editor: Hossein Jafari

Copyright (c) 2014 A. Atangana and S. C. Oukouomi Noutchie. This is an open access article distributed under the Creative Commons Attribution License, which permits unrestricted use, distribution, and reproduction in any medium, provided the original work is properly cited.

A novel approach is proposed to deal with a class of nonlinear partial equations including integer and noninteger order derivative. This class of equations cannot be handled with any other commonly used analytical technique. The proposed method is based on the multi-Laplace transform. We solved as an example some complicated equations. Three illustrative examples are presented to confirm the applicability of the proposed method. We have presented in detail the stability, the convergence and the uniqueness analysis of some examples.

\section{Introduction}

In order to investigate the present and future behaviors of a physical problem, many scholars always convert the observed fact into mathematical formula. In the recent decade it was observed that many physical problems were described with partial differential equation with either integer order derivative or fractional order derivative. Some of these equations can be classified as ordinary (linear or nonlinear) partial (linear or nonlinear) differential equations. The partial differential equations, both fractional and integer-order, have been documented as an overriding modeling technique particularly in the last few decades [1-5]. To accurately replicate the nonlocal, frequency-, and history-dependent properties of power law phenomena, some different modeling tools based on fractional operators have to be introduced.

However, as soon as this conversion is done, the next challenge is to find the solutions to these equations. Many scholars have developed method to show the existence and the uniqueness of the solution of these equations [6-8]. But when we are dealing with real world fact, one needs to find approximate, numerical, or exact solution of these equations in order to predict and analyze the solution as function of time and space and this renders the existence concept useless [9-11]. It is perhaps important to recall that finding the exact solution implies the existence of a solution and this is more convenient than just to provide a proof of the existence without presenting the solution. In order to be more practical, several methods have been proposed to find solutions to these equations. In the case of linear equations, some techniques using integral transform such as Mellin transform, Laplace transform, the Fourier transform, and the Sumudu transform, as well as other recent techniques, were proposed. In the case of nonlinear partial differential equations, asymptotic methods are dealing with equations with small parameters, perturbation methods are dealing with multilayers problems, and for some strong linearity, iterations methods such as homotopy perturbation, Adomian's decomposition, homotopy decomposition, variational iteration, and many others have been documented and proven efficient with limitations [12-17]. In 2011, Khan showed that it was possible to make use of the Laplace transform to actually derive solution of nonlinear equations. In his method, he coupled the Laplace series with the Poincare 
series [16]. Others adapted this method using the Sumudu transform and the idea of the Lagrange multiplier.

The question that remains is what will happen if a nonlinear partial differential is made up with mixed derivative only? Can these commonly used methods be suitable in finding the approximate or exact solution? The answer is perhaps no. One of the purposes of this paper is to present a novel or extended method that will be used to handle this class of partial derivative. The method makes use of the double Laplace transform and the Poincare series. Without loss of generality, the general form of this class of equation is given as follows:

$$
\begin{aligned}
\partial_{x^{n}}^{n} \partial_{y^{m}}^{m} & \cdots \partial_{t^{i}}^{i}[U(x, y, \ldots, t)] \\
= & L[U(x, y, \ldots, t)]+N[U(x, y, \ldots, t)] \\
& +f(x, y, \ldots, t)
\end{aligned}
$$

where $m, n, \ldots, i$ are integer and noninteger numbers, $L$ and $N$ are linear and nonlinear operators with only mixed derivative, respectively, and $f$ is a known function. However, to be more practical we will only consider the dimension to be two or three. The rest of the paper is presented as follows. The methodology of the technique is presented in Section 2. Application of the method with some examples is presented in Section 3. The stability, convergence, and uniqueness analysis will be presented in Section 4, and finally a conclusion is reached in Section 5.

\section{Methodology}

We devote this section to the discussion underpinning the methodology of the technique for solving (1). Nevertheless, before we present this methodology we will first present some properties of Laplace transform for integer and noninteger order.

Definition 1. The Laplace transform is a widely used integral transform with many applications in physics and engineering. The Laplace transform of the function $f$ is defined as follows:

$$
\mathscr{L}(f(x))(s)=\int_{0}^{\infty} e^{-s x} f(x) d x .
$$

2.1. Properties of Fractional Calculus. Two properties of the Laplace transform can be used to define the fractional integral operator as follows $[18,19]$ :

$$
\begin{gathered}
\mathscr{L}(I(f))=\mathscr{L}\left(\int_{0}^{x} f(\tau) d \tau\right)(s)=\frac{1}{s} \mathscr{L}(f(x))(s), \\
\mathscr{L}\left(I^{2}(f)\right)=\mathscr{L}\left(\int_{0}^{x} \int_{0}^{x_{1}} f(\tau) d \tau d x_{1}\right)(s) \\
=\frac{1}{s^{2}} \mathscr{L}(f(x))(s) .
\end{gathered}
$$

Now using the recursive method, we arrive at the following:

$$
\mathscr{L}\left(I^{n}(f)\right)=\frac{1}{s^{n}} \mathscr{L}(f(x))(s) .
$$

From the above equation we can obtain

$$
\begin{aligned}
\mathscr{L}\left(I^{n}(f)\right) & =\frac{1}{s^{n}} \mathscr{L}(f(x))(s) \\
& \Longrightarrow I^{n}(f)=\mathscr{L}^{-1}\left(\frac{1}{s^{n}} \mathscr{L}(f(x))(s)\right) .
\end{aligned}
$$

It is well known from the convolution theorem of Laplace transform that

$$
\mathscr{L}(f * g(x))(s)=\mathscr{L}(f(x))(s) \cdot \mathscr{L}(g(x))(s) .
$$

Now from the above formula if we chose $g(x)=x^{\alpha-1}$, with the information of (4), the fractional integral operator can be defined as follows:

$$
\begin{aligned}
{ }_{a} D_{t}^{\alpha} f(x)=\frac{1}{\Gamma(\alpha)} & \mathscr{L}^{-1}(\mathscr{L}(f(x))(s) \cdot \mathscr{L}(g(x))(s)) \\
{ }_{a} D_{t}^{\alpha} f(x) & =\frac{1}{\Gamma(\alpha)}(f * g(x)) \\
& =\frac{1}{\Gamma(\alpha)} \int_{a}^{x}(x-t)^{\alpha-1} f(t) d t
\end{aligned}
$$

Let us observe that Laplace transform of the fractional derivative with both Riemann-Liouville and Caputo is as follows:

$$
\begin{aligned}
& \mathscr{L}\left({ }_{0}^{C} D_{t}^{\alpha} f(x)\right)(s) \\
& \quad=s^{\alpha} F(s)-\sum_{k=0}^{n-1} s^{\alpha-k-1} f^{(k)}(0), \quad(n-1<\alpha \leq n) .
\end{aligned}
$$

Caputo use the usual initial conditions or values of the functions. On the other hand, we have the Riemann-Liouville; that is,

$$
\begin{array}{r}
\mathscr{L}\left({ }_{0} D_{t}^{\alpha} f(x)\right)(s)=s^{\alpha} F(s)-\sum_{k=0}^{n-1} s^{\alpha-k-1}{ }_{a} D_{t}^{p-k-1} f(0), \\
(n-1 \leq \alpha<n) .
\end{array}
$$

The above make use of the unusual initial value or conditions of the function; therefore, it is not suitable for the real world problems [19].

With the above references we will present the methodology of the technique. The first step in this technique is to apply on both sides of (1) the multi-Laplace transform to obtain

$$
\begin{gathered}
s_{1}^{m} s_{2}^{n} \cdots s_{i}^{i} u\left(s_{1}, s_{2}, \ldots, s_{i}\right)-F\left(s_{1}, s_{2}, \ldots, s_{i}, x, y, \ldots, t\right) \\
=\mathscr{L}_{x, y, \ldots, t}[L[U(x, y, \ldots, t)]+N[U(x, y, \ldots, t)] \\
+f(x, y, \ldots, t)]\left(s_{1}, s_{2}, \ldots, s_{i}\right) .
\end{gathered}
$$


We can now divide both sides by $s_{1}^{m} s_{2}^{n} \cdots s_{i}^{i}$ and apply the inverse Laplace transform on both sides to obtain

$$
\begin{aligned}
& U(x, y, \ldots, t) \\
& =g(x, y, \ldots, t) \\
& =\mathscr{L}_{x, y, \ldots, t}^{-1}\left[\frac{1}{s_{1}^{m} s_{2}^{n} \cdots s_{i}^{i}}\right. \\
& \quad \times\left(\mathscr{L}_{x, y, \ldots, t}[L[U(x, y, \ldots, t)]\right. \\
& \quad+N[U(x, y, \ldots, t)] \\
& \quad+f(x, y, \ldots, t)] \\
& \left.\left.\quad \times\left(s_{1}, s_{2}, \ldots, s_{i}\right)\right)\right](x, y, \ldots, t) .
\end{aligned}
$$

At this stage two iteration formulae can be developed, the first one using the idea of Lagrange multiplier and the second one using the homotopy idea. If we use the idea of Lagrange multiplier, we have that the Lagrange multiplier in Laplace space is given as follows:

$$
\lambda\left(s_{1}, s_{2}, \ldots, s_{i}\right)=\frac{1}{s_{1}^{m} s_{2}^{n} \cdots s_{i}^{i}} .
$$

With the above Lagrange multiplier in hand, we proposed the general integration to be in the form of

$$
\begin{aligned}
& U_{0}(x, y, \ldots, t)=g(x, y, \ldots, t), \\
& U_{n+1}(x, y, \ldots, t) \\
& =U_{n}(x, y, \ldots, t) \\
& +\mathscr{L}_{x, y, \ldots, t}^{-1}\left[\frac{1}{s_{1}^{m} s_{2}^{n} \cdots s_{i}^{i}}\right. \\
& \times\left(\mathscr { L } _ { x , y , \ldots , t } \left[L\left[U_{n}(x, y, \ldots, t)\right]\right.\right. \\
& +N\left[U_{n}(x, y, \ldots, t)\right] \\
& +f(x, y, \ldots, t)] \\
& \left.\left.\times\left(s_{1}, s_{2}, \ldots, s_{i}\right)\right)\right](x, y, \ldots, t)
\end{aligned}
$$

and the approximate or special solution can be obtained as

$$
U(x, y, \ldots, t)=\lim _{n \rightarrow \infty} U_{n+1}(x, y, \ldots, t) .
$$

If we use the idea of homotopy, we will assume that the solution can be in the form of series as follows:

$$
U(x, y, \ldots, t)=\sum_{n=0}^{\infty} p^{n} U_{n}(x, y, \ldots, t)
$$

With replacing this expression in (11) and after comparing terms of the same power of $p$, as well as using the polynomial proposed in [15], we obtain

$$
\begin{aligned}
& U_{0}(x, y, \ldots, t)=g(x, y, \ldots, t), \\
& U_{1}(x, y, \ldots, t) \\
& =\mathscr{L}_{x, y, \ldots, t}^{-1}\left[\frac{1}{s_{1}^{m} s_{2}^{n} \cdots s_{i}^{i}}\right. \\
& \times\left(\mathscr { L } _ { x , y , \ldots , t } \left[L\left[U_{0}(x, y, \ldots, t)\right]\right.\right. \\
& \quad+N\left[U_{0}(x, y, \ldots, t)\right] \\
& \quad+f(x, y, \ldots, t)] \\
& \left.\left.\quad \times\left(s_{1}, s_{2}, \ldots, s_{i}\right)\right)\right](x, y, \ldots, t)
\end{aligned}
$$

and for $n \geq 2$, we have

$$
\begin{aligned}
U_{n+1}(x, y, \ldots, t) & \\
=\mathscr{L}_{x, y, \ldots, t}^{-1}\left[\frac{1}{s_{1}^{m} s_{2}^{n} \cdots s_{i}^{i}}\right. & \\
& \times\left(\mathscr { L } _ { x , y , \ldots , t } \left[L\left[U_{0}(x, y, \ldots, t)\right]\right.\right. \\
& \left.+\mathscr{H}_{n}[(x, y, \ldots, t)]\right] \\
& \left.\left.\times\left(s_{1}, s_{2}, \ldots, s_{i}\right)\right)\right](x, y, \ldots, t)
\end{aligned}
$$

with of course $\mathscr{H}_{n}[(x, y, \ldots, t)]$ the polynomial proposed in [15].

We will illustrate this method with some examples and this is done in the next section

\section{Application}

We present in this section the application of this extension by solving some nonlinear and linear partial differential equations with mixed derivative only.

Example 2. To illustrate these methods, let us consider the following simple linear equation:

$$
\partial_{x t} u(x, t)+u=0 .
$$

Making use of methodology 2 presented is Section 2, we have

$$
\begin{aligned}
u(x, t)= & u(x, 0)+u(0, t)-u(0,0) \\
& -\mathscr{L}_{x, t}^{-1}\left[\frac{1}{s v}\left(\mathscr{L}_{x, t}[u(x, t)](s, v)\right)\right](x, t)
\end{aligned}
$$

and the general iteration formula for this is given by

$$
\begin{gathered}
u_{0}(x, t)=u(x, 0)+u(0, t)-u(0,0), \\
u_{n+1}(x, t)=-\mathscr{L}_{x, t}^{-1}\left[\frac{1}{s v}\left(\mathscr{L}_{x, t}\left[u_{n}(x, t)\right](s, v)\right)\right](x, t) .
\end{gathered}
$$


Therefore, using the iteration formula, we obtain

$$
\begin{aligned}
& u_{0}(x, t)=\operatorname{Cosh}[x]+\operatorname{Cosh}[t]-1, \\
& u_{1}(x, t)=-\frac{1}{2} e^{-t} x+\frac{e^{t} x}{2}+\frac{1}{2} e^{-x} t\left(-1+e^{2 x}-2 e^{x} x\right) \text {, } \\
& u_{2}(x, t)=-\frac{x^{2}}{2}+\frac{1}{4} e^{-t} x^{2}+\frac{e^{t} x^{2}}{4} \\
& +\frac{1}{4} e^{-x} t^{2}\left(1-2 e^{x}+e^{2 x}-e^{x} x^{2}\right) \text {, } \\
& u_{3}(x, t)=-\frac{1}{12} e^{-t} x^{3}+\frac{e^{t} x^{3}}{12}-\frac{t x^{3}}{6} \\
& +\frac{1}{36} e^{-x} t^{3}\left(-3+3 e^{2 x}-6 e^{x} x-e^{x} x^{3}\right), \\
& u_{4}(x, t)=-\frac{x^{4}}{24}+\frac{1}{48} e^{-t} x^{4}+\frac{e^{t} x^{4}}{48}-\frac{t^{2} x^{4}}{48}+\frac{1}{576} e^{-x} t^{4} \\
& \times\left(12-24 e^{x}+12 e^{2 x}-12 e^{x} x^{2}-e^{x} x^{4}\right), \\
& u_{5}(x, t) \\
& =-\frac{1}{240} e^{-t} x^{5}+\frac{e^{t} x^{5}}{240}-\frac{t x^{5}}{120}-\frac{t^{3} x^{5}}{720} \\
& +\frac{e^{-x} t^{5}\left(-60+60 e^{2 x}-120 e^{x} x-20 e^{x} x^{3}-e^{x} x^{5}\right)}{14400}, \\
& u_{6}(x, t)=-\frac{x^{6}}{720}+\frac{e^{-t} x^{6}}{1440}+\frac{e^{t} x^{6}}{1440}-\frac{t^{2} x^{6}}{1440}-\frac{t^{4} x^{6}}{17280} \\
& +\left(e ^ { - x } t ^ { 6 } \left(360-720 e^{x}+360 e^{2 x}-360 e^{x} x^{2}\right.\right. \\
& \left.\left.-30 e^{x} x^{4}-e^{x} x^{6}\right) \times(518400)^{-1}\right), \\
& u_{7}(x, t)=-\frac{e^{-t} x^{7}}{10080}+\frac{e^{t} x^{7}}{10080}-\frac{t x^{7}}{5040}-\frac{t^{3} x^{7}}{30240}-\frac{t^{5} x^{7}}{604800} \\
& +\left(e ^ { - x } t ^ { 7 } \left(-2520+2520 e^{2 x}-5040 e^{x} x\right.\right. \\
& \left.-840 e^{x} x^{3}-42 e^{x} x^{5}-e^{x} x^{7}\right) \\
& \left.\times(25401600)^{-1}\right) \text {. }
\end{aligned}
$$

And then, the summation of the first 11 terms is given as

$$
\begin{aligned}
u[x, t]= & \sum_{n=0}^{10} u[x, t, n]=-1-\frac{e^{-t} x}{2}+\frac{e^{t} x}{2}-\frac{x^{2}}{2}+\frac{1}{4} e^{-t} x^{2} \\
& +\frac{e^{t} x^{2}}{4}-\frac{1}{12} e^{-t} x^{3}+\frac{e^{t} x^{3}}{12}-\frac{t x^{3}}{6}-\frac{x^{4}}{24} \\
& +\frac{1}{48} e^{-t} x^{4}+\frac{e^{t} x^{4}}{48}-\frac{t^{2} x^{4}}{48}-\frac{1}{240} e^{-t} x^{5}+\frac{e^{t} x^{5}}{240} \\
& -\frac{t x^{5}}{120}-\frac{t^{3} x^{5}}{720}-\frac{x^{6}}{720}+\frac{e^{-t} x^{6}}{1440}+\frac{e^{t} x^{6}}{1440}-\frac{t^{2} x^{6}}{1440}
\end{aligned}
$$

$$
\begin{aligned}
& -\frac{t^{4} x^{6}}{17280}-\frac{e^{-t} x^{7}}{10080}+\frac{e^{t} x^{7}}{10080}-\frac{t x^{7}}{5040}-\frac{t^{3} x^{7}}{30240} \\
& -\frac{t^{5} x^{7}}{604800}-\frac{x^{8}}{40320}+\frac{e^{-t} x^{8}}{80640}+\frac{e^{t} x^{8}}{80640}-\frac{t^{2} x^{8}}{80640} \\
& -\frac{t^{4} x^{8}}{967680}-\frac{t^{6} x^{8}}{29030400}-\frac{e^{-t} x^{9}}{725760}+\frac{e^{t} x^{9}}{725760} \\
& -\frac{t x^{9}}{362880}-\frac{t^{3} x^{9}}{2177280}-\frac{t^{5} x^{9}}{43545600} \\
& -\frac{t^{7} x^{9}}{1828915200}-\frac{x^{10}}{3628800}+\frac{e^{-t} x^{10}}{7257600} \\
& +\frac{e^{t} x^{10}}{7257600}-\frac{t^{2} x^{10}}{7257600}-\frac{t^{4} x^{10}}{87091200} \\
& -\frac{t^{6} x^{10}}{2612736000}-\frac{t^{8} x^{10}}{146313216000} \\
& +\frac{1}{2} e^{-x} t\left(-1+e^{2 x}-2 e^{x} x\right) \\
& +\frac{1}{4} e^{-x} t^{2}\left(1-2 e^{x}+e^{2 x}-e^{x} x^{2}\right) \\
& +\frac{1}{36} e^{-x} t^{3}\left(-3+3 e^{2 x}-6 e^{x} x-e^{x} x^{3}\right) \\
& +\frac{1}{576} e^{-x} t^{4}\left(12-24 e^{x}+12 e^{2 x}-12 e^{x} x^{2}-e^{x} x^{4}\right) \\
& +\left(e ^ { - x } t ^ { 5 } \left(-60+60 e^{2 x}-120 e^{x} x\right.\right. \\
& \left.\left.-20 e^{x} x^{3}-e^{x} x^{5}\right) \times(14400)^{-1}\right) \\
& +\left(e ^ { - x } t ^ { 6 } \left(360-720 e^{x}+360 e^{2 x}-360 e^{x} x^{2}\right.\right. \\
& \left.\left.-30 e^{x} x^{4}-e^{x} x^{6}\right) \times(518400)^{-1}\right) \\
& +\left(e ^ { - x } t ^ { 7 } \left(-2520+2520 e^{2 x}-5040 e^{x} x-840 e^{x} x^{3}\right.\right. \\
& \left.\left.-42 e^{x} x^{5}-e^{x} x^{7}\right) \times(25401600)^{-1}\right) \\
& +\left(e ^ { - x } t ^ { 8 } \left(20160-40320 e^{x}+20160 e^{2 x}\right.\right. \\
& -20160 e^{x} x^{2}-1680 e^{x} x^{4}-56 e^{x} x^{6} \\
& \left.\left.-e^{x} x^{8}\right) \times(1625702400)^{-1}\right) \\
& +\left(e ^ { - x } t ^ { 9 } \left(-181440+181440 e^{2 x}-362880 e^{x} x\right.\right. \\
& -60480 e^{x} x^{3}-3024 e^{x} x^{5}-72 e^{x} x^{7} \\
& \left.\left.-e^{x} x^{9}\right) \times(131681894400)^{-1}\right) \\
& +\left(e ^ { - x } t ^ { 1 0 } \left(1814400-3628800 e^{x}+1814400 e^{2 x}\right.\right. \\
& -1814400 e^{x} x^{2}-151200 e^{x} x^{4} \\
& \left.-5040 e^{x} x^{6}-90 e^{x} x^{8}-e^{x} x^{10}\right)
\end{aligned}
$$




$$
\begin{aligned}
& \left.\times(13168189440000)^{-1}\right) \\
+ & \operatorname{Cosh}[t]+\operatorname{Cosh}[x] .
\end{aligned}
$$

Realize that if $n$ is very large, then the solution of this equation is

$$
u[x, t]=\sum_{n=0}^{\infty} u_{n}(x, t)=\operatorname{Cosh}[x-t]
$$

This is the exact solution of our equation. We will examine the solution for the fractional version.

Example 3. Let us consider the following linear fractional differential equation:

$$
\partial_{x^{\alpha} t^{\beta}}^{\alpha+\beta} u(x, t)+u=0 .
$$

Making use of methodology 2 presented in Section 2, we have

$$
\begin{aligned}
u(x, t)= & u(x, 0)+u(0, t)-u(0,0) \\
& -\mathscr{L}_{x, t}^{-1}\left[\frac{1}{s^{\alpha} v^{\beta}}\left(\mathscr{L}_{x, t}[u(x, t)](s, v)\right)\right](x, t)
\end{aligned}
$$

and the general iteration formula for this is given by

$$
\begin{aligned}
& u_{0}(x, t)=u(x, 0)+u(0, t)-u(0,0), \\
& u_{n+1}(x, t)=-\mathscr{L}_{x, t}^{-1}\left[\frac{1}{s^{\alpha} v^{\beta}}\left(\mathscr{L}_{x, t}\left[u_{n}(x, t)\right](s, v)\right)\right](x, t) .
\end{aligned}
$$

Therefore, using the iteration formula we obtain

$$
\begin{aligned}
& u_{0}(x, t)=u(x, 0)+u(0, t)-u(0,0)=1, \\
& u_{1}(x, t)=\frac{t^{\beta} x^{\alpha}}{\Gamma[1+\alpha] \Gamma[1+\beta]}, \\
& u_{2}(x, t)=\frac{t^{2 \beta} x^{2 \alpha}}{\Gamma[1+2 \alpha] \Gamma[1+2 \beta]}, \\
& u_{3}(x, t)=\frac{t^{3 \beta} x^{3 \alpha}}{\Gamma[1+3 \alpha] \Gamma[1+3 \beta]}, \\
& u_{4}(x, t)=\frac{t^{4 \beta} x^{4 \alpha}}{\Gamma[1+4 \alpha] \Gamma[1+4 \beta]}, \\
& u_{n}(x, t)=\frac{t^{n \beta} x^{n \alpha}}{\Gamma[1+n \alpha] \Gamma[1+n \beta]} .
\end{aligned}
$$

It is very important to realize that if $n$ is very large, then the solution of this equation is

$$
u[x, t]=\sum_{n=0}^{\infty} u_{n}(x, t)=\sum_{n=1}^{\infty} \frac{t^{n \beta} x^{n \alpha}}{\Gamma[1+n \alpha] \Gamma[1+n \beta]} .
$$

This is the exact solution of our equation.
Example 4. Let us consider the following nonlinear partial differential equation :

$$
\begin{aligned}
\partial_{x t}^{2} u= & 2 u \partial_{x^{2} t^{2}}^{4} u+4 \partial_{x} u \partial_{x t^{2}}^{3} u+4 \partial_{x^{2} t}^{3} u \partial_{t} u \\
& +4\left(\partial_{x t}^{2} u\right)^{2}+\partial_{t^{2}}^{2} u \partial_{x^{2}}^{2} u+u^{2}+\partial_{x t^{2}}^{3} u^{3} .
\end{aligned}
$$

The above equation is very complicated due to the strong nonlinearity; we will therefore present a special solution to it by applying methodology two presented in Section 2 .

$$
\begin{aligned}
u(x, t)=u(x, 0)+u(0, t)- & u(0,0) \\
-\mathscr{L}_{x, t}^{-1}\left[\frac { 1 } { s v } \left(\mathscr{L}_{x, t}[\right.\right. & 2 u \partial_{x^{2} t^{2}}^{4} u+4 \partial_{x} u \partial_{x t^{2}}^{3} u \\
& +4 \partial_{x^{2} t}^{3} u \partial_{t} u+4\left(\partial_{x t}^{2} u\right)^{2} \\
& +\partial_{t^{2}}^{2} u \partial_{x^{2}}^{2} u+u^{2} \\
& \left.\left.\left.+\partial_{x t^{2}}^{3} u^{3}\right](s, v)\right)\right](x, t) .
\end{aligned}
$$

The second and the third steps produce

$$
\begin{aligned}
u_{0}(x, t)=u(x, 0)+u(0, t) & -u(0,0) \\
u_{1}(x, t)=-\mathscr{L}_{x, t}^{-1}\left[\frac { 1 } { s v } \left(\mathscr{L}_{x, t}\right.\right. & {\left[2 u_{0} \partial_{x^{2} t^{2}}^{4} u_{0}+4 \partial_{x} u_{0} \partial_{x t^{2}}^{3} u_{0}\right.} \\
& +4 \partial_{x^{2} t}^{3} u_{0} \partial_{t} u_{0}+4\left(\partial_{x t}^{2} u_{0}\right)^{2} \\
& +\partial_{t^{2}}^{2} u_{0} \partial_{x^{2}}^{2} u_{0}+u_{0}^{2} \\
& \left.\left.\left.+\partial_{x t^{2}}^{3} u_{0}^{3}\right](s, v)\right)\right](x, t) .
\end{aligned}
$$

And for any natural number greater than one we have

$$
\begin{aligned}
& u_{n}(x, t)=-\mathscr{L}_{x, t}^{-1}\left[\frac { 1 } { s v } \left(\mathscr{L}_{x, t}\right.\right. 2 H_{n}^{1}(x, t)+4 H_{n}^{2}(x, t) \\
&+4 H_{n}^{3}(x, t)+4 H_{n}^{4}(x, t) \\
&+H_{n}^{5}(x, t)+H_{n}^{6}(x, t) \\
&\left.\left.\left.+H_{n}^{7}(x, t)\right](s, v)\right)\right](x, t), \\
& H_{n}^{1}(x, t)=\sum_{j=0}^{n-1} u_{j}(x, t) \partial_{x^{2} t^{2}} u_{n-j}(x, t),
\end{aligned}
$$




$$
\begin{aligned}
& H_{n}^{2}(x, t)=\sum_{j=0}^{n-1} \partial_{x} u_{j}(x, t) \partial_{x t^{2}} u_{n-j}(x, t), \\
& H_{n}^{3}(x, t)=\sum_{j=0}^{n-1} \partial_{t} u_{j}(x, t) \partial_{x^{2} t} u_{n-j}(x, t), \\
& H_{n}^{4}(x, t)=\sum_{j=0}^{n-1} \partial_{x t} u_{j}(x, t) \partial_{x t} u_{n-j}(x, t), \\
& H_{n}^{5}(x, t)=\sum_{j=0}^{n-1} \partial_{t^{2}} u_{j}(x, t) \partial_{x^{2}} u_{n-j}(x, t), \\
& H_{n}^{6}(x, t)=\sum_{j=0}^{n-1} u_{j}(x, t) u_{n-j}(x, t), \\
& H_{n}^{7}(x, t)=\sum_{j=0}^{n-1} \sum_{k=0}^{j} \partial_{x t^{2}} u_{j}(x, t) \partial_{x t^{2}} u_{j-k}(x, t) \partial_{x t^{2}} \\
& \times u_{n-j-1}(x, t) .
\end{aligned}
$$

The above can be resumed in the following algorithm.

Algorithm 5. (i) Input: $J(x, t)$ as initial guest,

(ii) $j$-number terms in the rough calculation

(iii) Output: $u_{\text {approx }}(x, t)$, the approximate solution.

Step 1. Put $u_{0}(x, t)=u(x, 0)+u(0, t)-u(0,0)$ and $u_{\text {approx }}(x, t)=u_{0}(x, t)$.

Step 2. For $j=0$ to $n-1$ do Step 3, Step 4, and Step 5.

Step 3. Compute

$$
\begin{aligned}
& H_{n}^{1}(x, t)=\sum_{j=0}^{n-1} u_{j}(x, t) \partial_{x^{2} t^{2}} u_{n-j}(x, t), \\
& H_{n}^{2}(x, t)=\sum_{j=0}^{n-1} \partial_{x} u_{j}(x, t) \partial_{x t^{2}} u_{n-j}(x, t), \\
& H_{n}^{3}(x, t)=\sum_{j=0}^{n-1} \partial_{t} u_{j}(x, t) \partial_{x^{2} t} u_{n-j}(x, t), \\
& H_{n}^{4}(x, t)=\sum_{j=0}^{n-1} \partial_{x t} u_{j}(x, t) \partial_{x t} u_{n-j}(x, t), \\
& H_{n}^{5}(x, t)=\sum_{j=0}^{n-1} \partial_{t^{2}} u_{j}(x, t) \partial_{x^{2}} u_{n-j}(x, t), \\
& H_{n}^{6}(x, t)=\sum_{j=0}^{n-1} u_{j}(x, t) u_{n-j}(x, t),
\end{aligned}
$$

$$
\begin{gathered}
H_{n}^{7}(x, t)=\sum_{j=0}^{n-1} \sum_{k=0}^{j} \partial_{x t^{2}} u_{j}(x, t) \partial_{x t^{2}} u_{j-k}(x, t) \partial_{x t^{2}} \\
\times u_{n-j-1}(x, t) .
\end{gathered}
$$

Step 4. Compute

$$
\begin{aligned}
u_{n+1}(x, t)=u_{n}(x, t) & \\
=-\mathscr{L}_{x, t}^{-1}\left[\frac { 1 } { s v } \left(\mathscr{L}_{x, t}[\right.\right. & H_{n}^{1}(x, t)+4 H_{n}^{2}(x, t) \\
& +4 H_{n}^{3}(x, t)+4 H_{n}^{4}(x, t) \\
& +H_{n}^{5}(x, t)+H_{n}^{6}(x, t) \\
& \left.\left.\left.+H_{n}^{7}(x, t)\right](s, v)\right)\right](x, t) .
\end{aligned}
$$

Step 5. Compute $u_{\text {approx }}(x, t)=u_{\text {approx }}(x, t)+u_{n+1}(x, t)$

Stop.

We will present in the next section the analysis of convergence and uniqueness of the especial solution of (29) for using method 2 .

\section{Convergence and Uniqueness Analysis}

The reason of this part is to demonstrate in detail the convergence and the uniqueness of the nonlinear equation while using the proposed iteration method; we will therefore consider the following equation:

$$
\begin{aligned}
\partial_{x t}^{2} u= & 2 u \partial_{x^{2} t^{2}}^{4} u+4 \partial_{x} u \partial_{x t^{2}}^{3} u+4 \partial_{x^{2} t}^{3} u \partial_{t} u \\
& +4\left(\partial_{x t}^{2} u\right)^{2}+\partial_{t^{2}}^{2} u \partial_{x^{2}}^{2} u+u^{2}+\partial_{x t^{2}}^{3} u^{3}
\end{aligned}
$$

Think about the Hilbert space $\mathscr{H}=L^{2}((\eta, \lambda) \times[0, T])$ defined as

$$
\begin{aligned}
& \mathscr{H}=\{(u, v):(\eta, \lambda) \times[0, T] \text { with, } \\
&\left.\mathscr{L}_{x, t}^{-1}\left[\frac{1}{s v}\left(\mathscr{L}_{x, t}[u(x, t)](s, v)\right)\right](x, t)<\infty\right\} .
\end{aligned}
$$

Then the operator is of the form

$$
\begin{aligned}
K(u)= & \partial_{x t}^{2} u=2 u \partial_{x^{2} t^{2}}^{4} u+4 \partial_{x} u \partial_{x t^{2}}^{3} u+4 \partial_{x^{2} t}^{3} u \partial_{t} u \\
& +4\left(\partial_{x t}^{2} u\right)^{2}+\partial_{t^{2}}^{2} u \partial_{x^{2}}^{2} u+u^{2}+\partial_{x t^{2}}^{3} u^{3}
\end{aligned}
$$

The proposed analytical technique is convergent if the subsequent necessities are met.

Hypothesis 1. It is likely for us to establish a positive constant, say $P$, such that the inner product holds in $\mathscr{H}$ :

$$
(K(u)-K(v), u-v) \leq P\|u-v\|, \quad \forall v, u \in \mathscr{H} .
$$


Hypothesis 2. As far as for all $v, u \in$ Hare bounded, this implies, we can find a positive constant say $D$ such that $\|u\|,\|v\| \leq D$; then, we can find $\Phi(D)>0$ such that

$$
(K(u)-K(v), g) \leq \Phi(D)\|u-v\|\|g\|, \quad \forall g \in H .
$$

We can accordingly declare the consequential theorem for the sufficient condition for the convergence of (41).

Theorem 6. Let us think about

$$
\begin{aligned}
K(u)= & 2 u \partial_{x^{2} t^{2}}^{4} u+4 \partial_{x} u \partial_{x t^{2}}^{3} u+4 \partial_{x^{2} t}^{3} u \partial_{t} u \\
& +4\left(\partial_{x t}^{2} u\right)^{2}+\partial_{t^{2}}^{2} u \partial_{x^{2}}^{2} u+u^{2}+\partial_{x t^{2}}^{3} u^{3}
\end{aligned}
$$

and think about the initial and boundary condition for (41); then, the proposed technique shows the way to a particular solution of (41).

We will present the proof of this theorem by just verifying Hypotheses 1 and 2.

Proof. Using the definition of our operator $K$, we have the following:

$$
\begin{aligned}
& K(u)-K(v)=2 u \partial_{x^{2} t^{2}}^{4} u+4 \partial_{x} u \partial_{x t^{2}}^{3} u+4 \partial_{x^{2} t}^{3} u \partial_{t} u \\
& +4\left(\partial_{x t}^{2} u\right)^{2}+\partial_{t^{2}}^{2} u \partial_{x^{2}}^{2} u+u^{2}-v^{2} \\
& +\partial_{x t^{2}}^{3}\left(u^{3}-v^{3}\right)-2 v \partial_{x^{2} t^{2}}^{4} v-4 \partial_{x} v \partial_{x t^{2}}^{3} v \\
& -4 \partial_{x^{2} t}^{3} v \partial_{t} v-4\left(\partial_{x t}^{2} v\right)^{2}-\partial_{t^{2}}^{2} v \partial_{x^{2}}^{2} v \\
& K(u)-K(v)=\partial_{x t}^{2}(u-v)+u^{2}-v^{2} \\
& +\partial_{x t^{2}}^{3}\left(u^{3}-v^{3}\right) \\
& +2 \partial_{x}\left(u \partial_{x t^{2}}^{3} u+\partial_{x t}^{2} u \partial_{t} u\right. \\
& \left.+\partial_{t x}^{2} u \partial_{t} u+\partial_{x} u \partial_{t^{2}}^{2} u\right) \\
& -2 \partial_{x}\left(v \partial_{x t^{2}}^{3} v+\partial_{x t}^{2} v \partial_{t} v\right. \\
& \left.+\partial_{t x}^{2} v \partial_{t} v+\partial_{x} v \partial_{t^{2}}^{2} v\right) \\
& K(u)-K(v)=\partial_{x t}^{2}(u-v)+\left(u^{2}-v^{2}\right) \\
& +\partial_{x t^{2}}^{3}\left(u^{3}-v^{3}\right)+\partial_{x^{2} t^{2}}^{4} u-\partial_{x^{2} t^{2}}^{4} v, \\
& K(u)-K(v)=\partial_{x t}^{2}(u-v)+\left(u^{2}-v^{2}\right) \\
& +\partial_{x t^{2}}^{3}\left(u^{3}-v^{3}\right)+\partial_{x^{2} t^{2}}^{4}\left(u^{2}-v^{2}\right) .
\end{aligned}
$$

With the above reduction in hand, it is therefore possible for us to evaluate the following inner product:

$$
\begin{aligned}
(K(u) & -K(v),(u-v)) \\
= & \left(\left(u^{2}-v^{2}\right), u-v\right)+\left(\partial_{x t^{2}}^{3}\left(u^{3}-v^{3}\right), u-v\right) \\
& +\left(\partial_{x^{2} t^{2}}^{4}\left(u^{2}-v^{2}\right), u-v\right) .
\end{aligned}
$$

We will examine case after case and start with the high nonlinear part.

Take for granted that $u, v$ are bounded; consequently, we can find a positive constant $M$ such that $(u, u),(v, v)<M^{2}$. It follows by the use of Schwartz inequality that

$$
\left(\partial_{x t^{2}}^{3}\left(u^{3}-v^{3}\right), u-v\right) \leq\left\|\partial_{x t^{2}}^{3}\left(u^{3}-v^{3}\right)\right\|\|u-v\| .
$$

However, we can find a positive constant $\theta_{1}$ such that $\| \partial_{t^{2}}^{2}\left(u^{3}-\right.$ $\left.v^{3}\right)\left\|\leq \theta_{1}\right\| \partial_{t^{2}}^{3}\left(u^{3}-v^{3}\right) \|$ it follows from equation (43) that

$$
\left(\partial_{x t^{2}}^{2}\left(u^{3}-v^{3}\right), u-v\right) \leq \theta_{1} \theta_{2} \theta_{3}\left\|u^{3}-v^{3}\right\|\|u-v\| .
$$

We will recall that

$$
u^{3}-v^{3}=(u-v)\left(u^{2}+u v+v^{2}\right) .
$$

Thus

$$
\begin{aligned}
\left\|u^{3}-v^{3}\right\| & =\left\|(u-v)\left(u^{2}+u v+v^{2}\right)\right\| \\
& =\|(u-v)\|\left\|u^{2}+u v+v^{2}\right\| .
\end{aligned}
$$

Now making use of the triangular inequality together with the fact that $u$ and $v$ are bounded, we arrive at the following result:

$$
\left\|u^{3}-v^{3}\right\| \leq 6 M^{3}
$$

It follows that

$$
\left(\partial_{x t^{2}}^{3}\left(u^{3}-v^{3}\right), u-v\right) \leq 6 \theta_{1} \theta_{2} \theta_{3} M^{3}\|u-v\| .
$$

Also we have the following inequality:

$$
\begin{aligned}
\left(u^{2}-v^{2}, u-v\right) & \leq\left\|u^{2}-v^{2}\right\|\|u-v\| \\
& \leq 4 M^{2}\|u-v\| .
\end{aligned}
$$

We moreover have also that the Cauchy-SchwarzBunyakovsky inequality yields

$$
\begin{aligned}
& \partial_{x^{2} t^{2}}^{4}\left(\left(u^{2}-v^{2}\right), u-v\right) \\
& \quad \leq \theta_{4} \theta_{5} \theta_{6} \theta_{7}\left\|u^{2}-v^{2}\right\|\|u-v\| .
\end{aligned}
$$

Obviously due to the fact that it is possible for us to find two positive constants $\theta_{3}, \theta_{4}$ such that

$$
\begin{aligned}
& \partial_{x^{2} t^{2}}^{4}\left(\left(u^{2}-v^{2}\right), u-v\right) \\
& \quad \leq \theta_{4} \theta_{5}\left\|\left(u^{2}-v^{2}\right)_{x t}\right\|\|u-v\|,
\end{aligned}
$$

we can find another set of positive constants $\theta_{6} \theta_{7}$ in respect of the following inequality:

$$
\left\|\left(u^{2}-v^{2}\right)_{x t}\right\| \leq \theta_{6} \theta_{7}\left\|u^{2}-v^{2}\right\|
$$

and finally using the fact that $u$ and $v$ are bounded we obtain

$$
\partial_{x^{2} t^{2}}^{4}\left(\left(u^{2}-v^{2}\right), u-v\right) \leq 4 M^{2} \theta_{4} \theta_{5} \theta_{6} \theta_{7}\|u-v\| .
$$


Now substituting (51), (44), and (49) into (42) we arrive at the following:

$$
\begin{aligned}
& (K(u)-K(v),(u-v)) \\
& \quad \leq\left(6 \theta_{1} \theta_{2} \theta_{3} M^{3}+4 M^{2}+4 M^{2} \theta_{4} \theta_{5} \theta_{6} \theta_{7}\right)\|u-v\| .
\end{aligned}
$$

Take here

$$
P=6 \theta_{1} \theta_{2} \theta_{3} M^{3}+4 M^{2}+4 M^{2} \theta_{4} \theta_{5} \theta_{6} \theta_{7} .
$$

And then, Hypothesis 1 is verified. We will now verify Hypothesis 2, and to do this we quickly compute the relation as follows:

$$
\begin{aligned}
(K(u)-K(v), g)= & \left(\left(u^{2}-v^{2}\right), g\right)+\left(\partial_{x t^{2}}^{3}\left(u^{3}-v^{3}\right), g\right) \\
& +\left(\partial_{x^{2} t^{2}}^{4}\left(u^{2}-v^{2}\right), g\right) .
\end{aligned}
$$

Now using the Schwartz inequality and the fact that $u$ and $v$ are bounded, we obtain the following:

$$
\begin{aligned}
& (K(u)-K(v), g) \\
& \quad \leq\left(2 D+3 D^{2} \theta_{1} \theta_{2} \theta_{3}+2 D \theta_{4} \theta_{5} \theta_{6} \theta_{7}\right)\|u-v\|\|g\| .
\end{aligned}
$$

We will then consider $\Phi(D)=\left(2 D+3 D^{2} \theta_{1} \theta_{2} \theta_{3}+2 D \theta_{4} \theta_{5} \theta_{6} \theta_{7}\right)$ and Hypothesis 2 is also verified. We can therefore conclude that the proposed method works for (23). Our next concern is to establish the uniqueness of the special solution. This can be resumed in the following theorem.

Theorem 7. Taking into account the initial conditions for (23) then the special solution of (23) $u_{\text {esp }}$ to whom $u$ convergence is unique.

Proof. Assuming that we can find another special solution, say $v_{\text {esp }}$, then by making use of the inner product, we have

$$
\left(u_{\text {esp }}-v_{\text {esp }}, u_{\text {esp }}-v_{\text {esp }}\right) \leq 2 D\left\|u_{\text {esp }}-v_{\text {esp }}\right\| .
$$

Now if $u$ is the exact solution of (23), then according to Theorem 6 we can find suitable natural number $n$ and $m$ such that $\left\|u_{\text {esp }}-u\right\|<\varepsilon / 4 D$ and $\left\|u-v_{\text {esp }}\right\|<\varepsilon / 4 D$ with $\varepsilon$ a very small number closest to zero. Now taking the $\max (m, n)$, then

$$
\left(u_{\text {esp }}-v_{\text {esp }}, u_{\text {esp }}-v_{\text {esp }}\right) \leq \varepsilon .
$$

However according to the properties of the inner product,

$$
\begin{array}{r}
\left(u_{\text {esp }}-v_{\text {esp }}, u_{\text {esp }}-v_{\text {esp }}\right)=0 \\
\text { implies } u_{\text {esp }}-v_{\text {esp }}=0
\end{array}
$$

and Theorem 7 is verified.

\section{Conclusion}

Although many methods have been developed to deal with the existence and the uniqueness of some class of differential equations, there are still a large number of equations for which these methods cannot be used and even it is not possible to prove the existence of their solution, for instance, the Navier-Stock equation. However, it is important to point out that solving an equation analytically and finding the exact solution are more than proving the existence of this solution. The question that arises at this level is that should we perhaps focus on developing analytical methods that can be used to solve these complicated equations? Or should we perhaps focus on developing methods to prove their existence? We are afraid to reveal that for those dealing with real world problems the answer will be as follows: let us instead focus on developing methods to find analytical solutions of these equations since we need them to predict the future behaviour of these physical phenomena. Numerical methods can also be used for this purpose [20]. The main aim of this paper was to propose a method that can be used to solve a class of partial differential equations that other commonly used methods, such as the normal Laplace transform method, the Fourier method, the Sumudu method, the Green function method, and the Mellin transform method, as well as the recent developed iteration methods, cannot handle. We therefore presented some examples. We have with great success presented the stability, the convergence, and the uniqueness analysis. We presented this for both fractional and ordinary partial differential equations. The method proposed here makes use of the Laplace transform.

\section{Conflict of Interests}

The authors declare that there is no conflict of interests regarding the publication of this paper.

\section{References}

[1] J. A. Barker, "A generalized radial flow model for hydraulic tests in fractured rock," Water Resources Research, vol. 24, no. 10, pp. 1796-1804, 1988.

[2] J. Bear, Dynamics of Fluids in Porous Media, American Elsevier Environmental Science Series, Elsevier, New York, NY, USA, 1972.

[3] I. Podlubny, Fractional Differential Equations, vol. 198 of Mathematics in Science and Engineering, Academic Press, San Diego, Calif, USA, 1999.

[4] J. F. Botha and A. Cloot, "A generalised groundwater flow equation using the concept of non-integer order derivatives," Water SA, vol. 32, no. 1, pp. 1-7, 2006.

[5] A. Atangana and P. D. Vermeulen, "Analytical solutions of a space-time fractional derivative of groundwater flow equation," Abstract and Applied Analysis, vol. 2014, Article ID 381753, 11 pages, 2014.

[6] R. P. Agarwal, Y. Zhou, and Y. He, "Existence of fractional neutral functional differential equations," Computers \& Mathematics with Applications, vol. 59, no. 3, pp. 1095-1100, 2010.

[7] D. Baleanu, R. P. Agarwal, H. Mohammadi, and S. Rezapour, "Some existence results for a nonlinear fractional differential equation on partially ordered Banach spaces," Boundary Value Problems, vol. 2013, article 112, 2013.

[8] Y.-K. Chang and J. J. Nieto, "Some new existence results for fractional differential inclusions with boundary conditions," 
Mathematical and Computer Modelling, vol. 49, no. 3-4, pp. 605609, 2009.

[9] A. Atangana and S. C. Oukouomi Noutchie, "A modified groundwater flow model using the space time RiemannLiouville fractional derivatives approximation," Abstract and Applied Analysis, vol. 2014, Article ID 498381, 7 pages, 2014.

[10] A. Atangana, "Drawdown in prolate spheroidal-spherical coordinates obtained via Green's function and perturbation methods," Communications in Nonlinear Science and Numerical Simulation, vol. 19, no. 5, pp. 1259-1269, 2014.

[11] J.-H. Moon and B.-G. Lee, "Modeling and sensitivity analysis of a pneumatic vibration isolation system with two air chambers," Mechanism and Machine Theory, vol. 45, no. 12, pp. 1828-1850, 2010.

[12] J. H. He and F. J. Liu, "Local fractional variational iteration method for fractal heat transfer in silk cocoon hierarchy," Nonlinear Science Letters A, vol. 4, no. 1, pp. 15-20, 2013.

[13] H. Jafari and S. Seifi, "Homotopy analysis method for solving linear and nonlinear fractional diffusion-wave equation," Communications in Nonlinear Science and Numerical Simulation, vol. 14, no. 5, pp. 2006-2012, 2009.

[14] V. Daftardar-Gejji and H. Jafari, "Adomian decomposition: a tool for solving a system of fractional differential equations," Journal of Mathematical Analysis and Applications, vol. 301, no. 2, pp. 508-518, 2005.

[15] A. Atangana and A. Secer, "The time-fractional coupledKorteweg-de-Vries equations," Abstract and Applied Analysis, vol. 2013, Article ID 947986, 8 pages, 2013.

[16] Y. Khan and Q. Wu, "Homotopy perturbation transform method for nonlinear equations using He's polynomials," Computers \& Mathematics with Applications, vol. 61, no. 8, pp. 19631967, 2011.

[17] Y. Khan, "A method for solving nonlinear time-dependent drainage model," Neural Computing and Applications, vol. 23, no. 2, pp. 411-415, 2013.

[18] A. A. Kilbas, H. M. Srivastava, and J. J. Trujillo, Theory and Applications of Fractional Differential Equations, vol. 204 of North-Holland Mathematics Studies, Elsevier, Amsterdam, The Netherlands, 2006.

[19] A. Atangana and A. Secer, "A note on fractional order derivatives and table of fractional derivatives of some special functions," Abstract and Applied Analysis, vol. 2013, Article ID 279681, 8 pages, 2013.

[20] A. H. Bhrawy and M. M. Al-Shomrani, "A Jacobi dual-Petrov Galerkin-Jacobi collocation method for solving Korteweg-de Vries equations," Abstract and Applied Analysis, vol. 2012, Article ID 418943, 16 pages, 2012. 


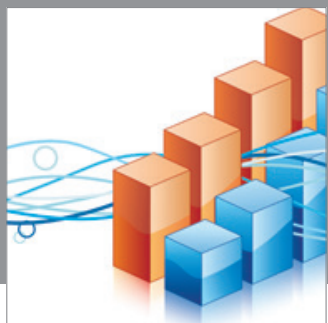

Advances in

Operations Research

mansans

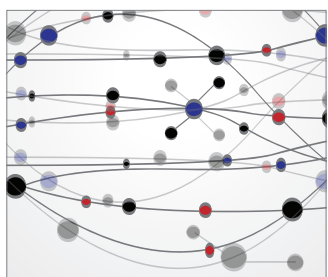

The Scientific World Journal
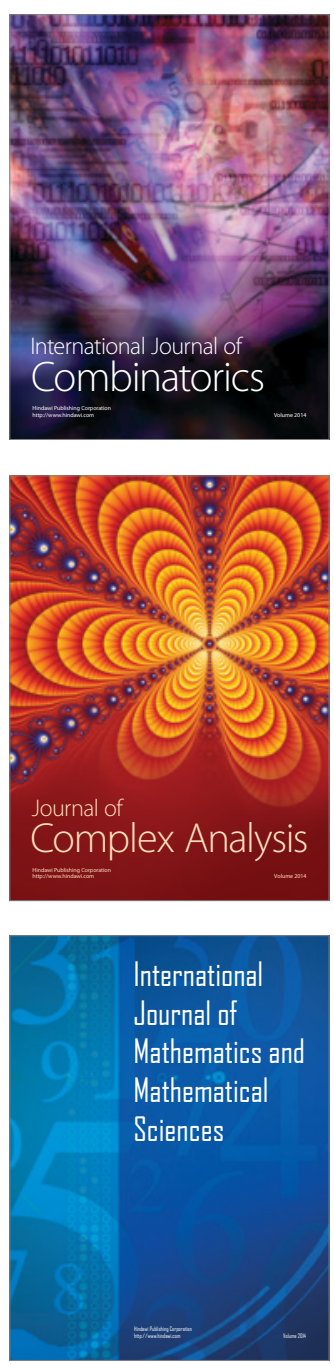
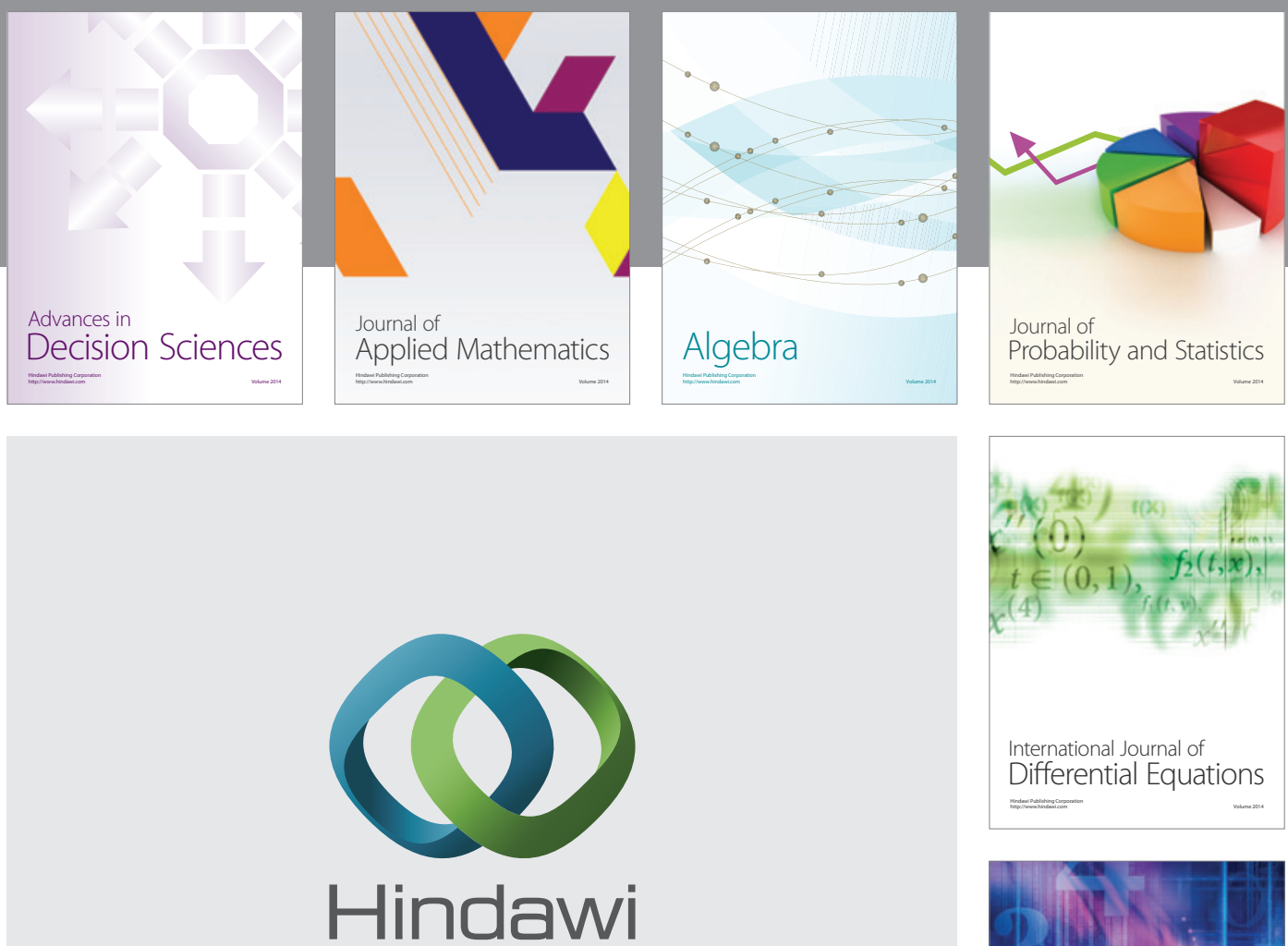

Submit your manuscripts at http://www.hindawi.com
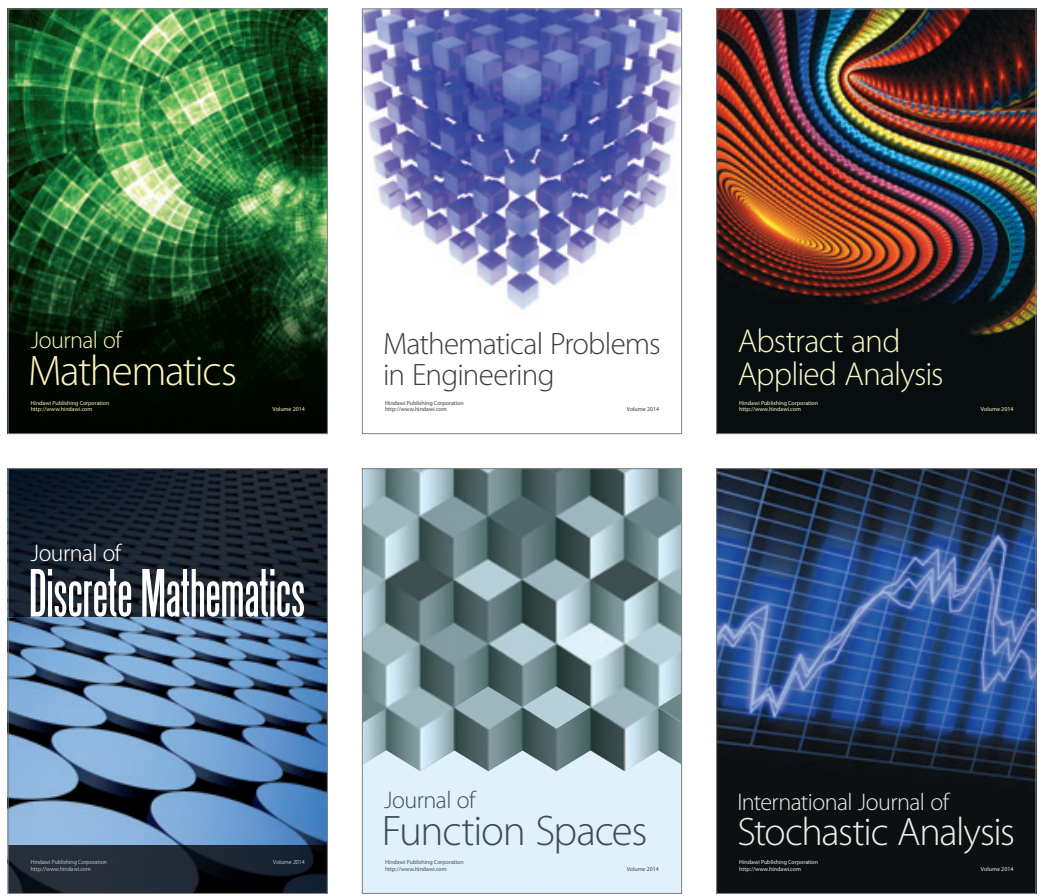

Journal of

Function Spaces

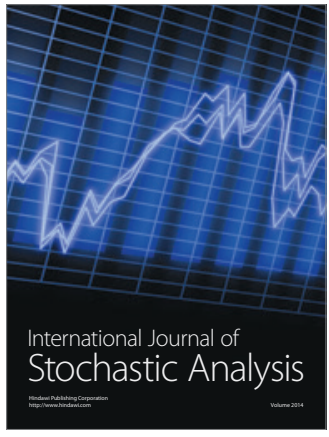

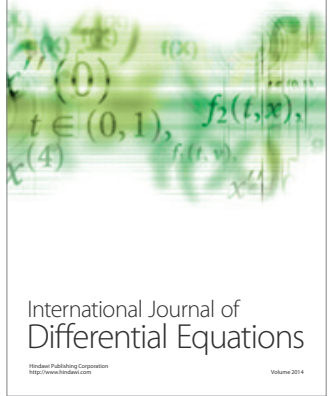
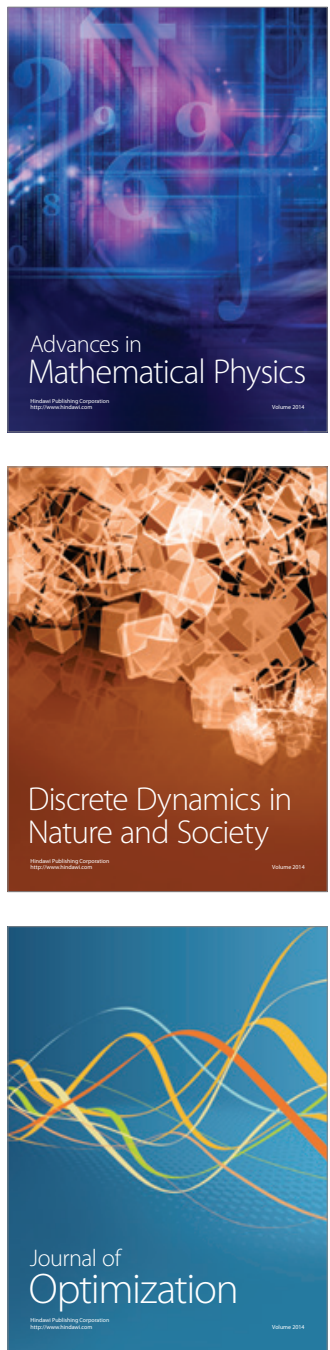